\title{
High glucose induces activation of the local renin-angiotensin system in glomerular endothelial cells
}

\author{
HUI PENG ${ }^{1 *}$, YAN-FANG XING ${ }^{2 *}$, ZENG-CHUN YE $^{1}$, CAN-MING LI ${ }^{1}$, \\ PENG-LI LUO ${ }^{1}$, MING LI ${ }^{1}$ and TAN-QI LOU ${ }^{1}$

\begin{abstract}
${ }^{1}$ Department of Internal Medicine, Division of Nephrology, The Third Affiliated Hospital of Sun Yat-sen University, of Guangzhou Medical University, Guangzhou, Guangdong 510150, P.R. China
\end{abstract} \\ Guangzhou, Guangdong 510630; ${ }^{2}$ Department of Internal Medicine, Division of Nephrology, The Third Affiliated Hospital
}

Received May 24, 2013; Accepted November 19, 2013

DOI: $10.3892 / \mathrm{mmr} .2013 .1855$

\begin{abstract}
Activation of the intrarenal renin-angiotensin system (RAS), which has been identified in podocytes and mesangial cells, is a novel mechanism in the progression of diabetic kidney disease (DKD). The present study aimed to identify the local RAS in glomerular endothelial cells (GEnCs). Rat GEnCs were stimulated by culture medium containing $30 \mathrm{mmol} / 1$ glucose for 12, 24, 48 and $72 \mathrm{~h}$. Angiotensin II (Ang II) concentrations in cell lysates and culture media were examined by ELISA and mRNA levels of angiotensinogen and renin in cell lysates were analyzed by quantitative polymerase chain reaction. Ang II type 1 receptor (AT1R), Ang II type 2 receptor (AT2R), renin and angiotensinogen levels in cell lysates were determined by western blot analysis. Localization of intracellular AT1R, AT2R, angiotensinogen and renin was identified by confocal immunofluorescence microscopy. Consequently, high glucose (HG) increased intracellular and extracellular Ang II levels. Captopril and chymostatin (inhibitor of chymase, an enzyme that converts Ang I to Ang II) were able to antagonize HG-induced Ang II generation. Moreover, HG increased angiotensinogen production in GEnCs and reduced renin mRNA expression without altering renin protein production. However, HG decreased AT1R levels and resulted in AT2R shifting from the nuclear to perinuclear region in GEnCs. In conclusion, HG activated the intracellular RAS in rat GEnCs and the underlying mechanism may involve angiotensin-converting enzyme (ACE) and non-ACE pathways. The effects of HG on GEnCs may also involve the substrate and receptors of Ang II.
\end{abstract}

Correspondence to: Professor Tan-Qi Lou, Department of Internal Medicine, Division of Nephrology, The Third Affiliated Hospital of Sun Yat-sen University, 600 Tianhe Road, Guangzhou, Guangdong 510630, P.R. China

E-mail: lou.tq@163.com

*Contributed equally

Key words: angiotensin II, diabetic kidney disease, renin angiotensin, glomerular endothelial cell

\section{Introduction}

Numerous studies have demonstrated the existence of an independent renin-angiotensin system (RAS) in the kidney and described its role in diabetic kidney disease (DKD). Locally generated angiotensin II (Ang II) in the kidney has been confirmed to be an important factor that mediates the progression of DKD. In addition to its blood pressure regulating effects, Ang II induces a number of non-hemodynamic effects through growth factors, profibrogenic cytokines and even proinflammatory factors $(1,2)$. The benefits of angiotensin-converting enzyme inhibitors (ACEIs) or Ang II receptor blockers in DKD also reflect the importance of non-hemodynamic effects of Ang II in mediating DKD.

The location and generation of the intrarenal RAS are not clearly understood and Ang II levels in several intrarenal compartments have been shown to be higher than those found systemically (3). A number of studies have indicated that the intrarenal RAS is activated by hyperglycemia, resulting in local Ang II generation (4). In addition, previous studies have shown that a high glucose concentration (HG) activates the RAS in podocytes and mesangial cells $(5,6)$.

Glomerular endothelial cell (GEnC) injury may result in proteinuria and glomerular sclerosis (7) and induce a loss of the glomerular filtration rate $(8,9)$. The endothelin system has an important role in DKD (10). Systemic endothelial dysfunction is prominent in type I and II diabetes (11). Although the pathophysiological mechanisms of GEnC injury remain to be fully determined, increasing evidence has shown that Ang II mediates the injury (12). Pharmacological inhibition of Ang II may therefore reduce GEnC injury and apoptosis.

In the present study, it was hypothesized that $\mathrm{HG}$, a characteristic of the diabetic milieu, results in the activation of a local RAS in GEnCs and experiments were performed to delineate the pathways involved.

\section{Materials and methods}

Cell culture. The rat glomerular endothelial cells (GEnCs) were established and characterized as described previously by Adler (13). According to this method, we developed the rat GEnCs with qualified stability and reliability. We have 
completed a series of studies based on this cell line $(14,15)$. Thus, the rat GEnCs in the present study was justified and reliable. Cells were cultured in RPMI-1640 medium (Life Technologies, Carlsbad, CA, USA) containing 10\% fetal bovine serum (Life Technologies), 10\% Nu-serum (BD Biosciences, Franklin Lakes, NJ, USA) and $5 \mathrm{mM}$ D-glucose. Culture flasks were stored in a humidified environment at $37^{\circ} \mathrm{C}$ in an atmosphere of $95 \% \mathrm{O}_{2}$ and $5 \% \mathrm{CO}_{2}$. The medium was replaced every 36-48 $\mathrm{h}$. The confluent GEnCs, which were serum-deprived for $24 \mathrm{~h}$, were exposed to $\mathrm{HG}$ medium containing $30 \mathrm{mM}$ D-glucose for $12,24,48$ and $72 \mathrm{~h}$. To correct for hyperosmolarity, equivalent concentrations of mannitol were added to control sets of cells.

Ang II measurement. Ang II levels were determined in rat GEnC lysates and conditioned culture media. After rat GEnC seeding on 6-well plates and serum restriction for $24 \mathrm{~h}$, the media were changed as described above. The media were collected at 12, 24, 48 and $72 \mathrm{~h}$, the cells were washed with ice-cold PBS, scraped from the plates in the presence of extraction buffer ( $1 \mathrm{ml}$ lysis buffer, $10 \mu \mathrm{l}$ PMSF, $5 \mu 1$ phosphatase inhibitor, $1 \mu \mathrm{l}$ protease inhibitor and $200 \mu \mathrm{ul}$ extraction buffer for one plate; Nanjing KeyGen Biotech, Nanjing, China) and homogenized. The cell lysates were centrifuged at $14,000 \mathrm{x} \mathrm{g}$ for $15 \mathrm{~min}$ at $4^{\circ} \mathrm{C}$, supernatants were collected, protein concentrations were determined using a bicinchoninic acid protein assay kit (Nanjing KeyGen Biotech) and samples were adjusted to a final concentration of $0.5 \mathrm{ng} / \mu \mathrm{l}$ (16). Ang II levels in the cell lysates and culture media were measured by ELISA (USCN Life Science \& Technology, Missouri City, TX, USA) according to the manufacturer's instructions.

To determine whether the increase in Ang II generation induced by HG was dependent on ACE and/or non-ACE (i.e., chymase) pathways, GEnCs were incubated in the presence of the ACEI, captopril (1.0 mmol/l; Sigma-Aldrich, St. Louis, MO, USA) or chymase inhibitor, chymostatin $(50 \mu \mathrm{mol} / \mathrm{l}$; Sigma-Aldrich), for $60 \mathrm{~min}$ prior to the introduction of $\mathrm{HG}$. Protein harvesting and measurement of Ang II levels were performed as previously outlined.

Measurement of mRNA levels. The levels of renin and angiotensinogen mRNA expression were estimated by quantitative polymerase chain reaction (qPCR) analysis. Total-RNA was purified by the phenol and guanidine isothiocyanate cesium chloride method (TRIzol kit; Life Technologies). The RNA pellet was resuspended in RNase-free water. qPCR was performed utilizing commercially available primers (AGT: forward, 5'-GGCAAATCTGGGCAAGATGG-3'; reverse, 5'-GCTCGTAGATGGCGAACAGGA-3'; Renin: forward, 5'-AGGATCAGTGCTGAATGGGGTGA-3'; reverse, 5'-GGTTGTGAATCTCACAGGCAGTGT-3' and SYBR Premix Ex Taq II (Code, DRR081; Takara Biotechnology (Dalian) Co., Ltd., Dalian, China) for renin and angiotensinogen. Fluorescence for each cycle was quantitatively analyzed using the ABI Prism 7000 sequence detection system (Life Technologies). The results were reported as relative expression, normalized with GAPDH housekeeping gene as an endogenous control and expressed in arbitrary units.

Western blot analysis. GEnCs harvested from plates were lysed in extraction buffer (Nanjing KeyGen Biotech). Equal quantities $(20-40 \mu \mathrm{g})$ of protein were heated at $100^{\circ} \mathrm{C}$ for 5 min and electrophoresis was performed using a $10 \%$ acrylamide denaturing sodium dodecyl sulfate-polyacrylamide gel with 20-40 $\mu \mathrm{g}$ loaded per lane. Proteins were then transferred to separated polyvinylidene fluoride membranes (Pall Corporation, Port Washington, NY, USA), which were then incubated in blocking buffer A (1X PBS, $0.05 \%$ Tween-20 and $5 \%$ non-fat milk) for $1 \mathrm{~h}$ at room temperature, followed by overnight incubation at $4{ }^{\circ} \mathrm{C}$ with a $1: 400$ dilution of primary antibodies against Ang II type 1 and 2 receptors (AT1R and AT2R; Santa Cruz Biotechnology, Inc., Santa Cruz, CA, USA), anti-renin antibody (Santa Cruz Biotechnology, Inc.) and 1:2,000 dilution of anti-angiotensinogen antibody (Abcam, Cambridge, MA, USA), or polyclonal anti-GAPDH antibody (Proteintech Group, Chicago, IL, USA). The membranes were then washed twice for $7 \mathrm{~min}$ in $1 \mathrm{X}$ PBS with $0.05 \%$ Tween-20. Membranes were incubated for $1 \mathrm{~h}$ at room temperature in buffer A (1X PBS, $0.05 \%$ Tween-20 and 5\% non-fat milk) in which a 1:1,000 dilution of horseradish peroxidase-linked goat anti-rabbit immunoglobulin G (IgG; Proteintech Group) was added. Detection of specific protein bands was performed with chemiluminescence using the Enhanced Chemiluminescence Plus detection system (Millipore, Billerica, MA, USA) and recorded on X-ray film (Kodak, Rochester, NY, USA). Densitometric quantitation was performed using Quantity One GS-800 software (Bio-Rad, Hercules, CA, USA).

Immunofluorescence studies. GEnCs were seeded onto glass coverslips. Cells were fixed with $4 \%$ paraformaldehyde for $15 \mathrm{~min}$ at room temperature, permeabilized with $0.3 \%$ Triton solution, stained for anti-AT1R, anti-AT2R, anti-renin or anti-angiotensinogen antibodies overnight at $4{ }^{\circ} \mathrm{C}$ and incubated with Alexa 488-labeled goat anti-rabbit IgG (Invitrogen Life Technologies). Staining specificity was confirmed by omission of the primary antibody. Images were visualized under a confocal fluorescence microscope (magnification, x400; Zeiss Ikon, Dresden, Germany).

Statistical analysis. Values are expressed as the mean \pm standard error (SE) of the mean. Analysis of variance with a post hoc Tukey's test was used for statistical analysis. $\mathrm{P}<0.05$ was considered to indicate a statistically significant difference.

\section{Results}

HG increases intracellular and extracellular Ang II levels in GEnCs. After $12 \mathrm{~h}$ of incubation with HG, Ang II levels in the conditioned media of GEnCs were significantly increased compared with that of the normal glucose and osmotic groups $(n=3$ per group; $\mathrm{P}<0.05)$. However, Ang II levels in the conditioned media were similar in the normal glucose and osmotic groups and Ang II concentrations in cell lysates were almost equal in all three groups. When the exposure time was extended to $72 \mathrm{~h}$, significantly increased intracellular and extracellular Ang II levels were detected in the HG groups compared with that of the normal glucose and osmotic groups ( $\mathrm{n}=3$ per group; $\mathrm{P}<0.05)$. Ang II levels in conditioned media and cell lysates were comparable in the normal glucose and osmotic groups under extended incubation periods. However, when HG-stimulated cells were maintained for 24 or $48 \mathrm{~h}$, no significant variation 
in Ang II levels in cultured media or cell lysates were detected among the three groups. Therefore, cells were incubated with HG for 12 and $72 \mathrm{~h}$ in subsequent experiments (Fig. 1).

$H G$-induces increases in Ang II levels in GEnCs is dependent upon ACE and non-ACE pathways. As HG was found to increase Ang II generation in GEnCs, the importance of ACE in the induction of Ang II was further investigated. GEnCs were preincubated for $60 \mathrm{~min}$ in the presence of captopril prior to incubation in HG. The addition of captopril fundamentally reduced Ang II levels in conditioned media and cell lysates incubated for $72 \mathrm{~h}$ (Fig. 2), suggesting that HG induced Ang II production in GEnCs in part through the ACE pathway. However, this reduction was not observed in conditioned media or cell lysates when the incubation time was $12 \mathrm{~h}$.

Previous studies confirmed that intracellular Ang II was generated not only through the classical ACE pathway, but also through non-ACE pathways $(17,18)$, such as those involving chymase. Therefore, GEnCs were preincubated in chymostatin (an inhibitor of chymase, an enzyme that converts Ang I to Ang II) for 60 min prior to incubation in HG. Ang II levels in these conditioned media and cell lysates were reduced after $72 \mathrm{~h}$ of incubation (Fig. 2). Notably, this reduction was not observed in conditioned media or cell lysates when the incubation time was $12 \mathrm{~h}$. Additionally, neither captopril nor chymostatin affected Ang II production in the normal glucose group.

It was observed that HG-induced Ang II generation may have been antagonized by captopril or chymostatin when GEnCs were stimulated by HG for $72 \mathrm{~h}$, but not when they were incubated for $12 \mathrm{~h}$. Therefore, cells were stimulated with $\mathrm{HG}$ for $72 \mathrm{~h}$ in subsequent experiments.

$H G$ increases angiotensinogen production in GEnCs. Angiotensinogen (precursor of Ang II) mRNA expression was assessed by qPCR after incubating GEnCs in HG, revealing a significant 7-fold increase compared with that of the normal glucose group $(\mathrm{P}<0.05)$.

To confirm this increase in angiotensinogen, angiotensinogen protein production was measured by western blot analysis. Total protein was extracted from GEnCs exposed to HG or normal glucose for $72 \mathrm{~h}$. HG incubation resulted in a significant, $200 \%$ increase in angiotensinogen production in the GEnCs (P<0.05; Fig. 3). Moreover, confocal immunofluorescence microscopy revealed that angiotensinogen staining was concentrated predominantly around the cell nuclei and exposure to HG led to an increase in the intensity of angiotensinogen staining compared with exposure to normal glucose or mannitol.

$H G$ reduces renin $m R N A$ expression in GEnCs without altering protein production. Total-RNA was extracted by TRIzol and qPCR was performed. GEnCs cultured in HG for $72 \mathrm{~h}$ displayed a decline in renin mRNA expression levels compared with that of the normal glucose group $(\mathrm{P}<0.05)$. The influence of $\mathrm{HG}$ on renin protein expression in GEnCs was evaluated by western blot analysis. Total protein was extracted from GEnCs exposed to $\mathrm{HG}$ or normal glucose for $72 \mathrm{~h}$ and HG was found to have no effect on the renin protein level.

In addition, confocal immunofluorescence microscopy revealed that renin staining was concentrated predominantly

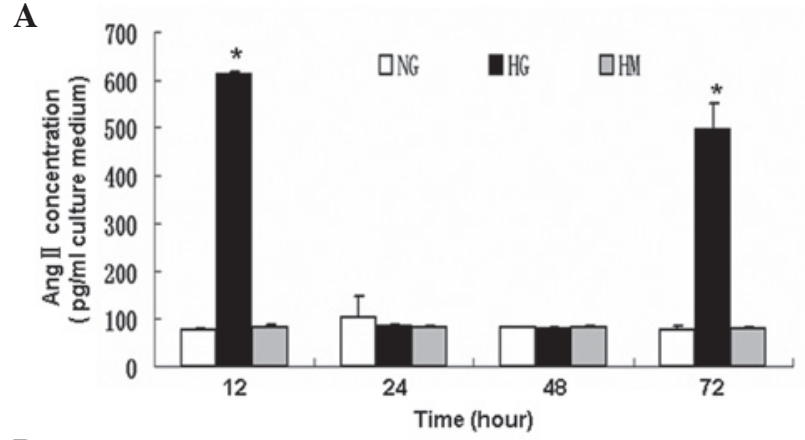

B

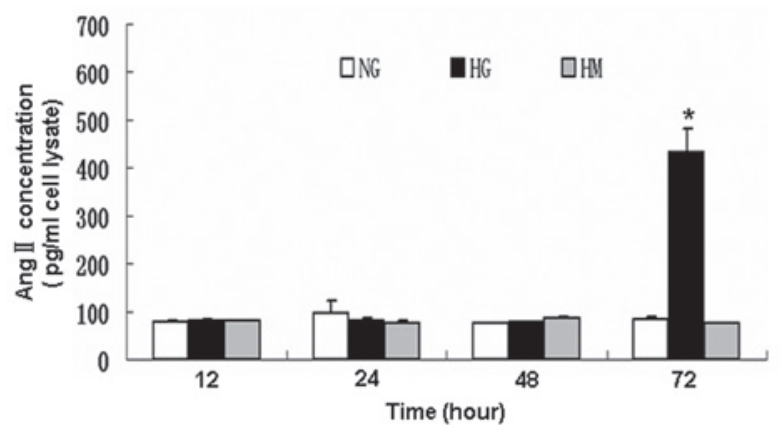

Figure 1. HG increased intracellular and extracellular Ang II levels. Ang II levels were detected (A) in the culture medium and (B) in the cell lysates under HG (30 mM D-glucose) for different time periods. Normal glucose (5 mM D-glucose) served as the control. Results are expressed as the mean \pm SE for three samples from each group. ${ }^{*} \mathrm{P}<0.05$ vs. the NG group. Ang II, angiotensin II; SE, standard error; NG, normal glucose; HG, high glucose; HM, normal glucose + hyperosmotic mannitol.
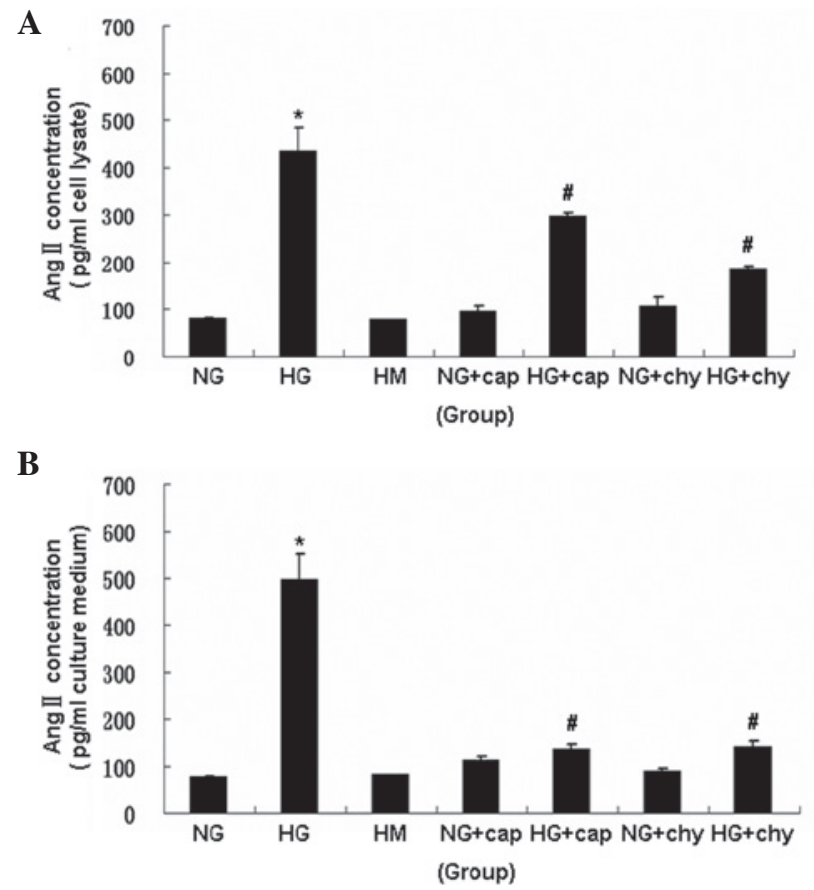

Figure 2. HG-induced increases of Ang II levels in glomerular endothelial cells depended on the ACE pathway and the non-ACE pathway. Glomerular endothelial cells were preincubated for $60 \mathrm{~min}$ in the presence of the ACE inhibitor or chymase inhibitor (chymostatin) prior to incubation with HG (30 mM). After $72 \mathrm{~h}$, Ang II levels were measured by ELISA (A) in the whole cell lysates and (B) in the cultured medium. Results are expressed as the mean \pm SE for three samples from each group. ${ }^{*} \mathrm{P}<0.05$ vs. the NG group; ${ }^{\#} \mathrm{P}<0.05$ vs. the HG group. Ang II, angiotensin II; ACE, angiotensin-converting enzyme; SE, standard error; NG, normal glucose; HG, high glucose; HM, normal glucose + hyperosmotic mannitol; cap, captopril; chy, chymostatin. 
A
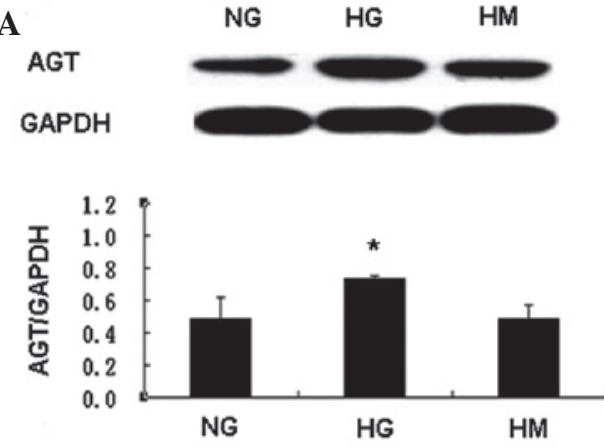

B

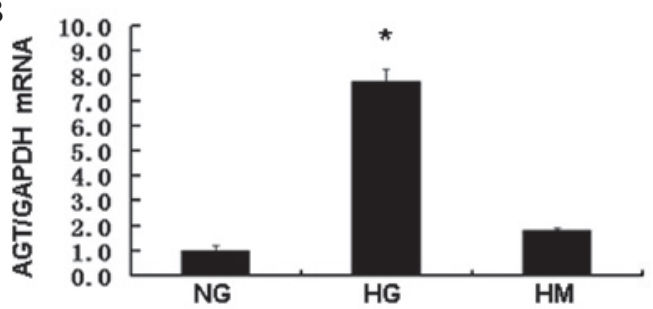

C

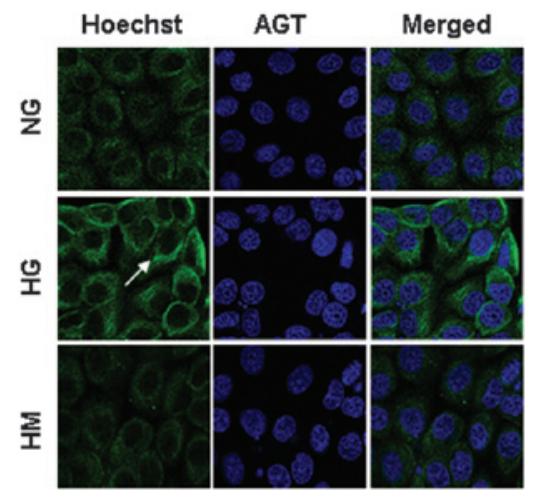

Figure 3. HG increased angiotensinogen mRNA and protein levels in glomerular endothelial cells. Quantitative polymerase chain reaction and western blot analysis were performed to detect the effects of $\mathrm{HG}$ on renin mRNA and protein expression. HG increased approximately (A) 2-fold in angiotensinogen protein expression and (B) 7-fold in angiotensinogen mRNA expression. (C) The indirect immunofluorescence results under confocal immunofluorescence microscopy conformed the change (white arrow shows cell nuclei). Results are expressed as the mean \pm standard error for three samples from each group. "P $<0.05$ vs. the NG group. HG, high glucose; NG, normal glucose; HM, normal glucose + hyperosmotic mannitol; AGT, angiotensin. Nuclei stained with Hoechst and cytoplasm stained with Alexa 488-labeled goat anti-rabbit IgG.

around cell nuclei, with marginal staining observed in the cytoplasm. The cellular distribution of renin was consistently unchanged following exposure to HG (Fig. 4).

$H G$ alters angiotensin receptor protein expression levels. AT1R and AT2R in GEnCs were detected by confocal immunofluorescence microscopy and western blot analysis. The AT1R is one of the best-elucidated angiotensin receptors and is responsible for numerous deleterious non-hemodynamic effects of Ang II on tissue injury (19). In the present study, exposure to $\mathrm{HG}$ resulted in a decline in the intensity of AT1R staining compared with exposure to normal glucose or mannitol. Western blot analysis of the cell lysates confirmed this result, demonstrating decreased levels of AT1R following exposure to HG (Fig. 5).
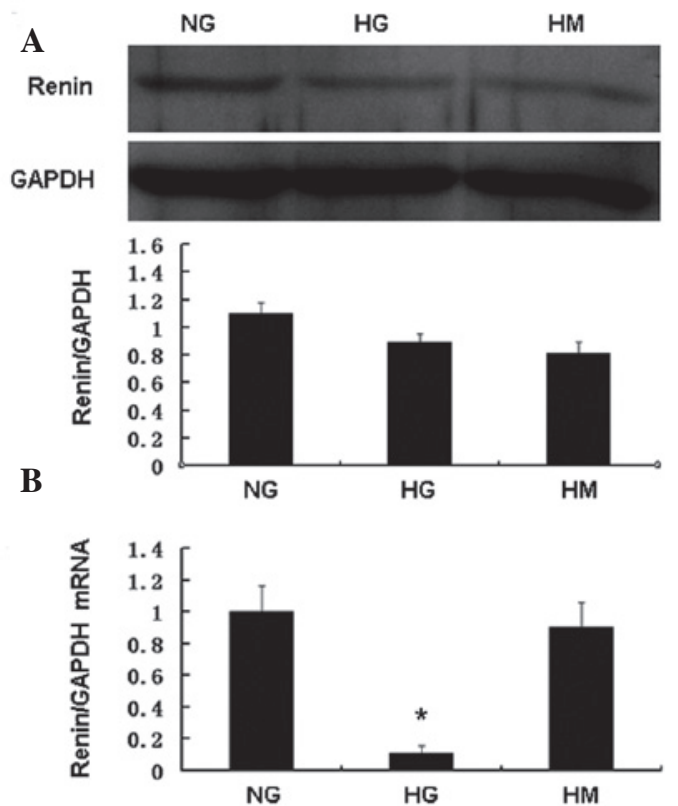

C

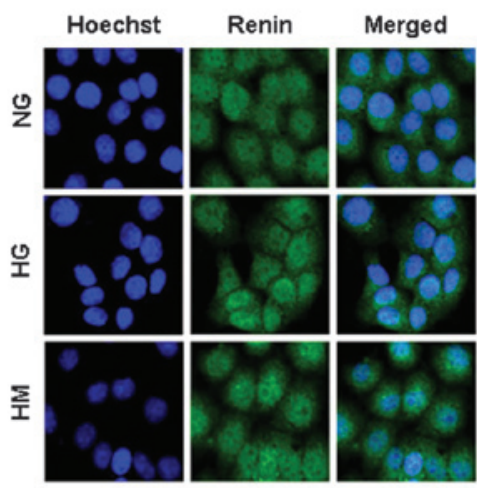

Figure 4. HG reduced renin mRNA expression in glomerular endothelial cells without alterating protein production. Quantitative polymerase chain reaction was performed to detect the effects of $\mathrm{HG}$ on renin mRNA. (B) HG resulted in $\sim 90 \%$ decrease in renin mRNA expression compared with that of the NG group $(\mathrm{P}<0.05)$. (A) The protein levels and (C) subcellular distribution of renin were unchanged following exposure to HG. Western blot analysis and confocal immunofluorescence microscopy were performed to detect the effects of glucose on protein expression and localization of renin. Results are expressed as the mean \pm standard error for three samples from each group. " $\mathrm{P}<0.05$ vs. the NG group. HG, high glucose; NG, normal glucose; HM, normal glucose + hyperosmotic mannitol. Nuclei stained with Hoechst and cytoplasm stained with Alexa 488-labeled goat anti-rabbit IgG.

The protein levels of AT2R, which mediates the protective effects of Ang II, were not changed by HG incubation. However, confocal immunofluorescence microscopy showed that the majority of AT2R staining was perinuclear in the HG group, whereas AT2R staining was observed in the nuclear and perinuclear regions in the normal glucose and mannitol groups (Fig. 6). Thus, AT1R and AT2R were localized inside GEnCs. HG affected the protein expression and localization of AT1R and the localization of AT2R.

\section{Discussion}

The present study identified an intracellular RAS in rat GEnCs, which was activated by HG. This activation may be involved in the progression of DKD. 
A

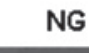

AT1

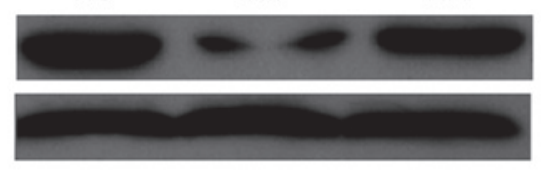

GAPDH

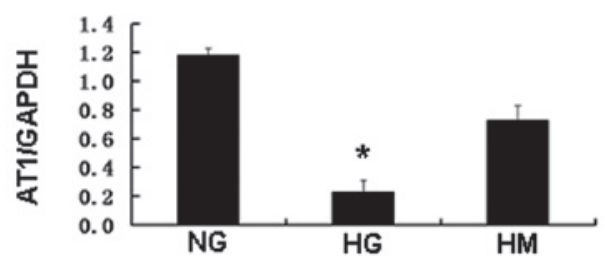

B

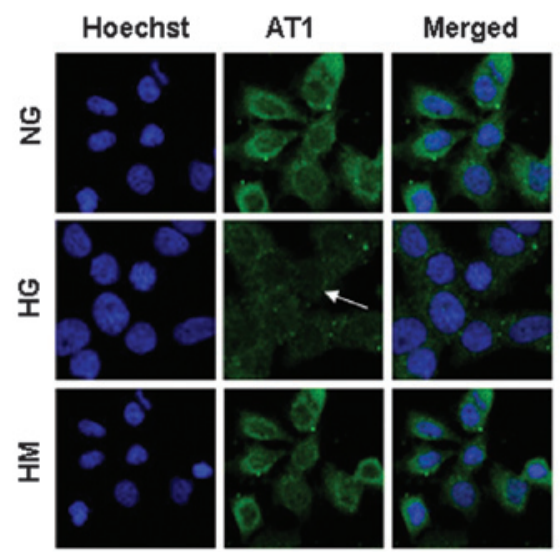

Figure 5. HG reduced AT1 protein in glomerular endothelial cells. Western blot analysis was performed to detect the effects of HG on AT1 protein. (A) HG resulted in $~ 85 \%$ decrease in AT1 protein expression compared with that of the NG group $(\mathrm{P}<0.05)$. (B) The indirect immunofluorescence results under confocal immunofluorescence microscopy confirmed the change (white arrow shows cell nuclei). Results are expressed as the mean \pm standard error for three samples from each group. ${ }^{*} \mathrm{P}<0.05$ vs. the NG group. HG, high glucose; AT1, angiotensin II type 1 receptor; NG, normal glucose; HM, normal glucose + hyperosmotic mannitol. Nuclei stained with Hoechst and cytoplasm stained with Alexa 488-labeled goat anti-rabbit IgG.

The classical RAS is characterized by the presence of RAS components, including angiotensinogen, conversion enzymes and the systemic synthesis of Ang II, which when binds with specific receptors results in a physiological response. However, a significant observation in DKD research was the existence of intracellular RASs in podocytes and mesangial cells, which were activated by HG $(5,6)$. Durvasula and Shankland (6) detected renin and AT1R in podocytes and Cristovam et al (5) detected ACE and chymase (an Ang II-generating enzyme) in mesangial cells. In addition, these two researcher groups identified that HG induced Ang II generation in cells. The intracellular production of Ang II stimulated growth in A7r5 vascular smooth muscle cells derived from fetal rat aorta and influenced mitosis in rat hepatoma cells $(20,21)$, which suggested its potential in mediating glomerular sclerosis. Thus, the intracellular RAS was characterized by the presence of RAS components inside the cell and synthesis of Ang II at an intracellular site (22). The results of the present study showed that Ang II was produced by rat GEnCs and that HG increased intracellular Ang II generation. The RAS components, including angiotensinogen, renin, AT1R and AT2R were detected in GEnCs; thus, the intracellular RAS may have
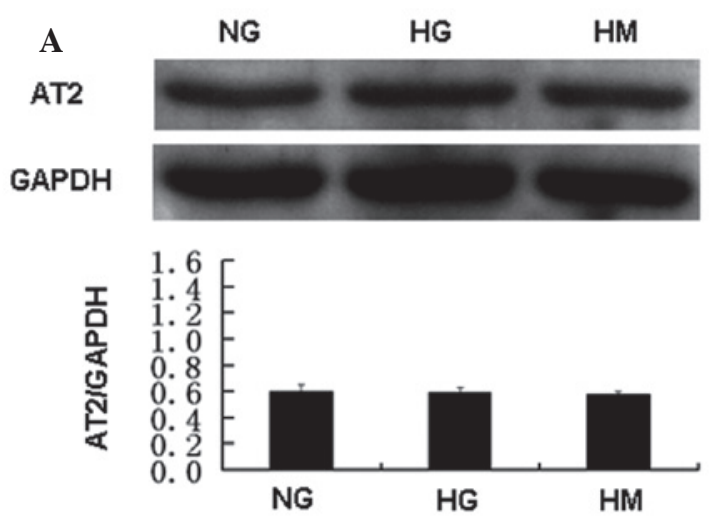

B

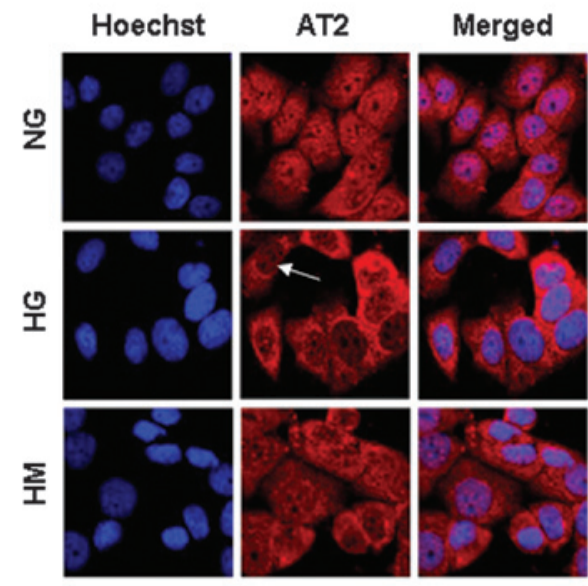

Figure 6. HG changed the localization of AT2 in glomerular endothelial cells. (A) Western blot analysis showed the protein levels of AT2 were unchanged following exposure to HG. (B) The majority of AT2 staining was perinuclear in the HG group (white arrow shows cell nuclei), whereas in the NG or HM groups AT2 staining was observed in the nuclear and perinuclear regions, as shown by confocal immunofluorescence microscopy. HG, high glucose; AT2, angiotensin II type 2 receptor; NG, normal glucose; HM, normal glucose + hyperosmotic mannitol. Nuclei stained with Hoechst and cytoplasm stained with Alexa 488-labeled goat anti-rabbit IgG.

be important in DKD. Consequently, the influence of $\mathrm{HG}$ on this specific system was examined.

Angiotensinogen, the precursor of Ang II, is generally produced by the liver under normal conditions and is secreted extracellularly due to the presence of a signal sequence and glycosylation. In this study, angiotensinogen production increased 2-fold in cell lysates when GEnCs were incubated with HG. This HG-induced increase in angiotensinogen was accompanied by a significant increase in angiotensinogen mRNA expression levels. These results suggested that HG facilitated the usability of a substrate for the final formation of Ang II in GEnCs. Increased angiotensinogen was also found in mesangial cells under HG conditions $(23,24)$. Additionally, a further study revealed that HG decreased extracellular secretion and increased intracellular retention of angiotensinogen in neonatal rat ventricular myocytes (25), which may be an alternative mechanism for the increase in intracellular angiotensinogen.

Moreover, the intracellular Ang II synthesis in rat GEnCs was detected and found that HG increased Ang II generation. Similarly, HG increased Ang II generation and expression in 
mesangial cells and podocytes, aggravating the progression of $\operatorname{DKD}(5,23,26)$. Intracellular Ang II has been considered to be a critical regulator of the local RAS $(27,28)$. Exposure to HG for $12 \mathrm{~h}$ induced an increase in extracellular, but not intracellular, Ang II levels. Notably, when the exposure time was extended to $72 \mathrm{~h}$, intracellular and extracellular Ang II levels increased. It is hypothesized that $\mathrm{HG}$ initially contributed to the increase in extracellular Ang II levels by stimulating GEnCs to secrete Ang II and then by accelerating Ang II production. Thus, the results of this study confirmed that $\mathrm{HG}$ induced the activation of an RAS in GEnCs.

Renin is a well-known enzyme that converts angiotensinogen to Ang I. Numerous studies have suggested that renin and/or its proenzyme precursor (prorenin) may interact with a tissue-specific receptor resulting in the progression of DKD (29). Intracellular renin may be derived locally and/or be absorbed from the circulation. In this study, confocal immunofluorescence microscopy showed that the majority of intracellular renin staining was nuclear and perinuclear. Together with the results of qPCR and western blot analysis targeting renin, a novel renin-producing cell was identified, the GEnC. Notably, HG induced a $90 \%$ reduction in renin mRNA expression compared with that of normal glucose. Ang II has previously been shown to inhibit renin synthesis and secretion, indicating the appearance of a physiologically important negative feedback control $(30,31)$. An additional study found that Ang II inhibited renin gene transcription via the protein kinase C (PKC) pathway (32). In the present study, western blot analysis demonstrated that renin levels were similar in cell lysates with and without HG interference. These results differed from those obtained by Durvasula and Shankland (6) in an investigation of renin production in podocytes; it was confirmed that HG increased renin mRNA and protein levels in cell lysates. Vidotti et al (24) obtained concurrent results, showing that HG incubation increased renin mRNA levels in mesangial cell lysates. These differences indicated the various roles of GEnCs and interstitial cells in the progression of DKD.

In addition to $\mathrm{ACE}$, the serine protease chymase, mainly expressed in mast cells, is increasingly recognized as a novel enzyme that converts Ang I to Ang II (33). Chymase is also expressed in the normal human kidney (5) and upregulated in DKD $(33,34)$. In the present study, the preincubation of rat GEnCs in the presence of the ACEI, captopril, or the chymase inhibitor, chymostatin, resulted in partial downregulation of HG-induced Ang II production in cell lysates and conditioned cultured media. Based on a previous study (34), it was hypothesized that HG increases Ang II generation in rat GEnCs via $\mathrm{ACE}$ and non-ACE pathways.

The Ang II receptors are a class of $\mathrm{G}$ protein-coupled receptors. According to the availability of non-peptide receptor ligands, Ang II receptors are divided into AT1R and AT2R groups. The AT1R is the most well-studied angiotensin receptor. Effects mediated by AT1R include vasoconstriction, aldosterone synthesis and secretion, increased vasopressin secretion, cardiac hypertrophy, decreased renal blood flow, renal tubular sodium reuptake and extracellular matrix formation. HG downregulated the density of AT1R via the protein kinase $\mathrm{C}$ pathway (35), which has an important role in the mechanisms by which HG affects the kidney. Consistent with previous findings, it was determined that exposure of GEnCs to HG decreased AT1R levels. Additionally, AT2R was found to increase the risk caused by Ang II, although the function of AT2R was not fully characterized. However, a previous study demonstrated that Ang II-mediated induction of nitric oxide (NO) was linked to nuclear AT2R, which is important in the maintenance of vascular tone. In the present study, HG induced a shift of AT2R from the nuclear to perinuclear region, which may weaken the NO pathway. Collectively, these findings suggest that AT1R and AT2R may mediate the adverse effects caused by the HG-activated intracellular RAS.

In conclusion, this study recognized an intracellular RAS in rat GEnCs, which may be activated by HG. The probable mechanisms may involve an increase in the substrates of angiotensinogen, ACE and non-ACE pathways, AT1R and AT2R regulation and renin feedback. However, the exact function and pathway of the intracellular RAS in DKD requires further investigation.

\section{Acknowledgements}

This study was supported by grants from the National Natural Science Funds of China (grant nos. 30800408 and 30771011).

\section{References}

1. Wolf G: Molecular mechanisms of angiotensin II in the kidney: emerging role in the progression of renal disease: beyond haemodynamics. Nephrol Dial Transplant 13: 1131-1142, 1998.

2. Suzuki Y, Ruiz-Ortega M, Lorenzo O, Ruperez M, Esteban V and Egido J: Inflammation and angiotensin II. Int J Biochem Cell Biol 35: 881-900, 2003.

3. Seikaly MG, Arant BS Jr and Seney FD Jr: Endogenous angiotensin concentrations in specific intrarenal fluid compartments of the rat. J Clin Invest 86: 1352-1357, 1990.

4. Zhang SL, To C, Chen X, et al: Effect of renin-angiotensin system blockade on the expression of the angiotensinogen gene and induction of hypertrophy in rat kidney proximal tubular cells. Exp Nephrol 9: 109-117, 2001.

5. Cristovam PC, Arnoni CP, de Andrade MC, et al: ACE-dependent and chymase-dependent angiotensin II generation in normal and glucose-stimulated human mesangial cells. Exp Biol Med (Maywood) 233: 1035-1043, 2008.

6. Durvasula RV and Shankland SJ: Activation of a local renin angiotensin system in podocytes by glucose. Am J Physiol Renal Physiol 294: F830-F839, 2008.

7. Lee LK, Meyer TW, Pollock AS and Lovett DH: Endothelial cell injury initiates glomerular sclerosis in the rat remnant kidney. J Clin Invest 96: 953-964, 1995.

8. Satchell SC and Tooke JE: What is the mechanism of microalbuminuria in diabetes: a role for the glomerular endothelium? Diabetologia 51: 714-725, 2008.

9. Ballermann BJ: Contribution of the endothelium to the glomerular permselectivity barrier in health and disease. Nephron Physiol 106: 19-25, 2007.

10. Zanatta CM, Canani LH, Silveiro SP, Burttet L, Nabinger G and Gross JL: Endothelin system function in diabetic nephropathy. Arq Bras Endocrinol Metabol 52: 581-588, 2008 (In Portuguese).

11. Jefferson JA, Shankland SJ and Pichler RH: Proteinuria in diabetic kidney disease: a mechanistic viewpoint. Kidney Int 74: 22-36, 2008.

12. Jaimes EA, Hua P, Tian RX and Raij L: Human glomerular endothelium: interplay among glucose, free fatty acids, angiotensin II, and oxidative stress. Am J Physiol Renal Physiol 298: F125-F132, 2010.

13. Adler S: Integrin receptors in the glomerulus: potential role in glomerular injury. Am J Physiol

14. Peng H, Luo P, Li Y, Wang C, Liu X, et al: Simvastatin Alleviates Hyperpermeability of Glomerular Endothelial Cells in Early-Stage Diabetic Nephropathy by Inhibition of RhoA/ROCK1. PLoS One 8: e80009, 2013. 
15. Peng $\mathrm{H}$, Wang $\mathrm{C}$, Ye ZC, et al: How increased VEGF induces glomerular hyperpermeability: a potential signaling pathway of Rac1 activation. Acta Diabetol 47 (Suppl 1): 57-63, 2010.

16. Durvasula RV, Petermann AT, Hiromura K, et al: Activation of a local tissue angiotensin system in podocytes by mechanical strain. Kidney Int 65: 30-39, 2004.

17. Li M,Liu K, Michalicek J, et al: Involvement of chymase-mediated angiotensin II generation in blood pressure regulation. J Clin Invest 114: 112-120, 2004.

18. Sadjadi J, Kramer GL, Yu CH, Burress Welborn M III, Chappell MC and Gregory Modrall J: Angiotensin converting enzyme-independent angiotensin ii production by chymase is up-regulated in the ischemic kidney in renovascular hypertension. J Surg Res 127: 65-69, 2005.

19. Homma T, Sonoda H, Manabe K, et al: Activation of renal angiotensin type 1 receptor contributes to the pathogenesis of progressive renal injury in a rat model for chronic cardiorenal syndrome. Am J Physiol Renal Physiol 302: F750-F761, 2012.

20. Filipeanu CM, Henning RH, de Zeeuw D and Nelemans A Intracellular Angiotensin II and cell growth of vascular smooth muscle cells. Br J Pharmacol 132: 1590-1596, 2001.

21. Cook JL, Zhang Z and Re RN: In vitro evidence for an intracellular site of angiotensin action. Circ Res 89: 1138-1146, 2001.

22. Kumar R, Singh VP and Baker KM: The intracellular renin-angiotensin system: a new paradigm. Trends Endocrinol Metab 18: 208-214, 2007.

23. Singh R, Singh AK, Alavi N and Leehey DJ: Mechanism of increased angiotensin II levels in glomerular mesangial cells cultured in high glucose. J Am Soc Nephrol 14: 873-880, 2003.

24. Vidotti DB, Casarini DE, Cristovam PC, Leite CA, Schor N and Boim MA: High glucose concentration stimulates intracellular renin activity and angiotensin II generation in rat mesangial cells. Am J Physiol Renal Physiol 286: F1039-F1045, 2004.

25. Singh VP, Le B, Bhat VB, Baker KM and Kumar R: High-glucose-induced regulation of intracellular Ang II synthesis and nuclear redistribution in cardiac myocytes. Am J Physiol Heart Circ Physiol 293: H939-H948, 2007.
26. Heikkila HM, Latti S, Leskinen MJ, Hakala JK, Kovanen PT and Lindstedt KA: Activated mast cells induce endothelial cell apoptosis by a combined action of chymase and tumor necrosis factor-alpha. Arterioscler Thromb Vasc Biol 28: 309-314, 2008.

27. Kumar R, Singh VP and Baker KM: The intracellular renin-angiotensin system: implications in cardiovascular remodeling. Curr Opin Nephrol Hypertens 17: 168-173, 2008.

28. Singh VP, Baker KM and Kumar R: Activation of the intracellular renin-angiotensin system in cardiac fibroblasts by high glucose: role in extracellular matrix production. Am J Physio Heart Circ Physiol 294: H1675-H1684, 2008.

29. Takahashi H, Ichihara A, Kaneshiro Y, et al: Regression of nephropathy developed in diabetes by (Pro)renin receptor blockade. J Am Soc Nephrol 18: 2054-2061, 2007.

30. Matsusaka T, Nishimura H, Utsunomiya $H$, et al: Chimeric mice carrying 'regional' targeted deletion of the angiotensin type $1 \mathrm{~A}$ receptor gene. Evidence against the role for local angiotensin in the in vivo feedback regulation of renin synthesis in juxtaglomerular cells. J Clin Invest 98: 1867-1877, 1996.

31. Schunkert H, Ingelfinger JR, Jacob H, Jackson B, Bouyounes B and Dzau VJ: Reciprocal feedback regulation of kidney angiotensinogen and renin mRNA expressions by angiotensin II. Am J Physiol 263: E863-E869, 1992

32. Muller MW, Todorov V, Kramer BK and Kurtz A: Angiotensin II inhibits renin gene transcription via the protein kinase $\mathrm{C}$ pathway. Pflugers Arch 444: 499-505, 2002.

33. Wasse H, Rivera AA, Huang R, et al: Increased plasma chymase concentration and mast cell chymase expression in venous neointimal lesions of patients with CKD and ESRD. Semin Dial 24: 688-693, 2011.

34. Huang XR, Chen WY, Truong LD and Lan HY: Chymase is upregulated in diabetic nephropathy: implications for an alternative pathway of angiotensin II-mediated diabetic renal and vascular disease. J Am Soc Nephrol 14: 1738-1747, 2003.

35. Amiri F and Garcia R: Regulation of angiotensin II receptors and PKC isoforms by glucose in rat mesangial cells. Am J Physiol 276: F691-F699, 1999. 